\title{
Que savent nos patients des antalgiques qu'ils consomment en automédication ? Etude prospective au sein de l'unité fonctionnelle des urgences odontologiques du Groupe Hospitalier Pitié-Salpêtrière.
}

\author{
Baaroun V, Mrejen N, Azerad J, Toledo R, Descroix V.
}

Service d'Odontologie, Groupe Hospitalier Pitié-Salpêtrière, 47-83 Boulevard de l'Hôpital, 75013, Paris, FRANCE

L'automédication est l'utilisation hors prescription médicale, par des personnes pour elles-mêmes ou pour leurs proches et de leur propre initiative, de médicaments considérés comme tels et ayant reçu I'AMM.

Cette pratique concerne entre 20 et $85 \%$ des français (Sofres 2001 ; Insee 2002 ; Afipa 2011). Parmi les médicaments d'automédication, le marché des antalgiques est particulièrement important : 5 des 10 spécialités les plus vendues en automédication en France sont des antalgiques (Afipa 2012). Cependant une partie de la population ignore les risques que peut engendrer cette pratique (surdosage, interaction médicamenteuse, non-respect des contre-indications...). Dans ce contexte, il est apparu intéressant de pouvoir étudier le recours en automédication aux médicaments antalgiques en cas de douleur dentaire. Cette étude a eu pour objectif principal d'évaluer les connaissances des patients à propos des médicaments qu'ils consomment en automédication.

Sur 132 patients interrogés consultant au sein de l'unité fonctionnelle des urgences odontologiques du Groupe Hospitalier Pitié-Salpêtrière, 103 avait consommé un antalgique en automédication avant leur venue. Le profil du « patient-type » était celui d'un homme d'environ 37 ans, avec un niveau bac, employé et bénéficiant de la Sécurité Sociale et d'une couverture complémentaire. Une nette majorité des patients $(72,5 \%)$ n'avait consommé qu'une seule molécule; il s'agissait du paracétamol pour $44 \%$, puis l'ibuprofène et l'aspirine. Les doses quotidiennes consommées étaient généralement inférieures aux posologies recommandées. Cependant, malgré ce sous-dosage, $63 \%$ des patients déclaraient un soulagement de la douleur, et ce indépendamment de la molécule utilisée.
Afin d'évaluer l'éventuel risque encouru par les patients, ceux-ci étaient interrogés sur leurs connaissances concernant la molécule consommée. Ainsi, il leur était demandé s'ils savaient ou non si l'antalgique consommé était dangereux pour le foie, l'estomac, chez la femme enceinte, et quelle était la posologie maximale à respecter. Une majorité de patients a déclaré ne pas savoir $(52,5 \%)$, et $34,3 \%$ ont répondu faux aux questions posées, amenant à plus de $80 \%$ la proportion de consommateurs d'antalgiques en automédication ignorant les potentiels risques qu'ils peuvent encourir. Malgré cela, $62,9 \%$ des patients se disaient prêts à renouveler cette pratique.

L'automédication, surtout antalgique, est pratiquée généralement pour " gagner du temps ", s'abstenir de consulter pour un symptôme jugé bénin, et parfois économiser le prix d'une consultation. Cependant, les publications concernant des accidents graves voire mortels liés à l'automédication se multiplient, et l'on sait désormais que la douleur dentaire est un facteur de risque de surdosage au paracétamol (Vogel et al. 2011). Malgré les nombreux avantages que représente l'automédication, notamment en termes d'économie de santé, les risques restent présents et inquiétants. Les fiches de bonnes pratiques de l'automédication de l'Afssaps et les campagnes réalisées en 2012 par le ministère de la santé ("Le médicament n'est pas un produit comme les autres ") visent à enrayer la banalisation de cette consommation pour aboutir à une depuis trop longtemps souhaitée « automédication responsable ».

BAAROUN Vanessa

vanessa.baaroun@gmail.com

This is an Open Access article distributed under the terms of the Creative Commons Attribution License 4.0, which permits unrestricted use, distribution, and reproduction in any medium, provided the original work is properly cited. 